\title{
Simulation of Storage Systems for increasing the Power Quality of Renewable Energy Sources
}

\author{
J. Mendez, A. Falcon and D. Hernandez \\ Institute of Intelligent Systems (SIANI) \\ Univ. of Las Palmas de Gran Canaria \\ Las Palmas, Spain \\ e-mail: jmendez@iusiani.ulpgc.es
}

\begin{abstract}
The use of renewable energy sources (RES) in electricity generation has many economical and environmental advantages, but has a downside in the instability and unpredictability introduced into the public electric systems. High variable energies such as wind power have a lack of stability and, to avoid short-term variations in power supplied to the grid, a local storage subsystem can be used to provide higher quality in the fed energy. This paper contains a mathematical model and a simulator focused on energy management that may be useful to evaluate the service quality, the energy efficiency and the required storage capacity.
\end{abstract}

\section{Key words}

Modelling Power Systems, Energy Storage, Power Quality, Renewable Energies, Systems Simulation.

\section{Introduction}

The more important renewable sources, wind and solar power, are mainly related to the weather in a local geographic area. However, the weather is a chaotic system with limited predictability. Many countries follow two trends in the development and planning of their public electric systems; the first is the increase in the generation power from RES and the second one is the transition to open electricity markets. These two trends have a common impact on the public grids, because they both increase the number of agents in the system and the level of uncertainty in the balance between generation and load.

The use of RES reduces the economic costs due to lesser fuel consumption, and also reduces the greenhouse gas emission. However, the access of more and bigger RES electricity producers can increase the risk of fail and decrease the service quality. That risk can be reduced by increasing the power reserve based on high response gradient systems. These, e.g. diesel or hydraulic, have a high speed of change in their generated power, that is suitable to balance the frequent sudden and unpredictable changes of RES-based electricity production. Therefore, the positive impact of the use of RES on the cost of fuel consumption would have a negative impact on the global cost of electricity systems.

The control and planning of public electric systems covers a widespread set of levels, ranging from the hundred millisecond domain associated to the frequency and voltage control[1], to the yearly planning domain. Precise regulations for these levels are the concern of the national Electricity Authorities of each country[2] as well as to supranational agencies. In each national system, the Transmission System Operator (TSO) deals with the management of the electric system in the different control and planning levels. With the increasing penetration of RES systems, the TSO becomes concerned with the impact on system stability[3]. The Electric Authorities of countries had included the power forecasting in its Regulatory Norms which goal is to preserve the quality of the electricity supply. The planning of an Electric System requires several levels related with different time scales as well as the weather forecasting. Regulation of power quality also requires several time scales. At very short time scale it is mainly related to voltage and frequency regulation, but at large time scale is mainly related to the power balance among the energy producers and the consumer loads. Different approaches related to power quality can be focused on different time scales as well as in different device details. Figure 1 illustrate the case 2 corresponding to this paper where we are interested in short term energy storage covering from few minutes to many weeks and in low device details. It means that this approach is mainly concerning to power and energy balance.

The forecasting of RES power production is a basic tool in the reduction of high operative reserve, which must be ready to be used. According to the practical experiences of E.ON, the largest German electric company, wind power is only as reliable as the weather forecasting [4]. If the wind power forecast differs from the actual infeed, the TSO must cover the difference by using the reserves, which must amount to $50-60 \%$ of the installed wind power. According to E.ON, the expected maximum forecast deviation is more important that the mean 
forecast error. This is because even if the actual infeed deviates from the forecast level on only a few days of the year, the TSO must also be prepared for this improbable eventuality and have sufficient capacity available, spinning reserve, for a reliable supply to still guaranteed and the correct balance between generation and load to be restored.

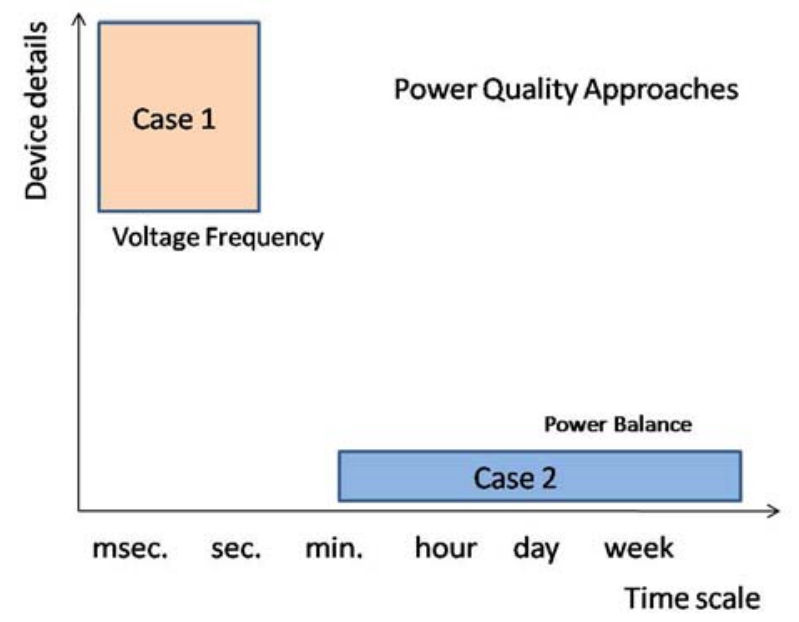

Fig. 1 Power quality approaches

This paper focuses on the modelling and simulation of energy storage systems coupled to RES producers to increase the quality of the energy feed to the public grids. Our approach is to study the energy and power management rather than the modelling and simulation associated with any specific technology, device or technical solution. We agree that a first level of a simulation can be a general one based on the power and energy flows and transfer, while a more detailed simulation of a defined solution, which must use defined models for wind farms[6] and grid interaction[7], can be achieved after the analysis of the results obtained in the power and energy oriented simulation. For example, a general simulation can provide the total amount of energy storage needed for an RES system based on its logged power data. At this stage, it does not matter which kind of technology is used in a more detailed forward modelling. This paper includes a mathematical model of power and energy transfer between the RES source, the energy storage and the public grid. A simulation based on Simulink is carried out and the results for different strategies and configurations are provided.

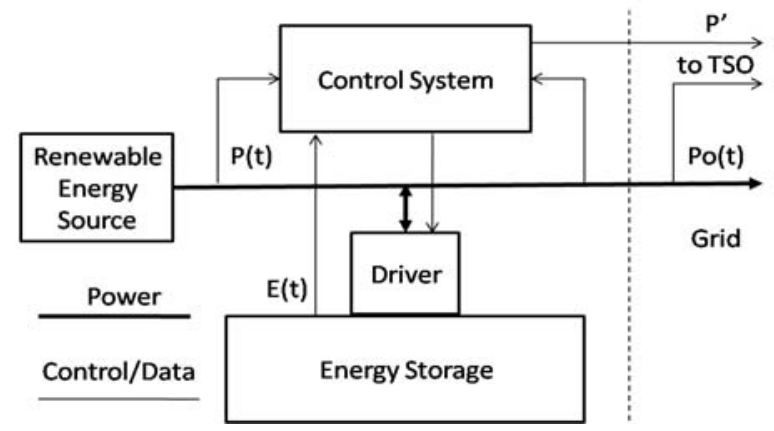

Fig. 2 Block diagram of the storage system to increase the quality of power fed to the grid.

\section{Mathematical Model}

The outline of the generic model of a RES producer coupled to a energy storage and connected to a public grid is shown in Figure 2. The RES provides a power $\mathrm{P}(\mathrm{t})$ that varies according the wind speed or sun radiation. The power planned to be sent to the grid in the hourly period is $\mathrm{P}^{\prime}$, its value had been computed by means of some forecasting procedure before being sent to the TSO. The power that the system is effectively sending to the grid is $\mathrm{Po}(\mathrm{t})$. The difference $\mathrm{Po}(\mathrm{t})-\mathrm{P}^{\prime}$ is the deviation between the planned and the fed power; this difference is logged by the measurement systems of the TSO and the control system. These values will provide some quality parameters that will reduce the economic billing of the RES producer. Although we have high quality prediction, the power of the RES is varying strongly in a hourly period as is illustrated in Figure 3.

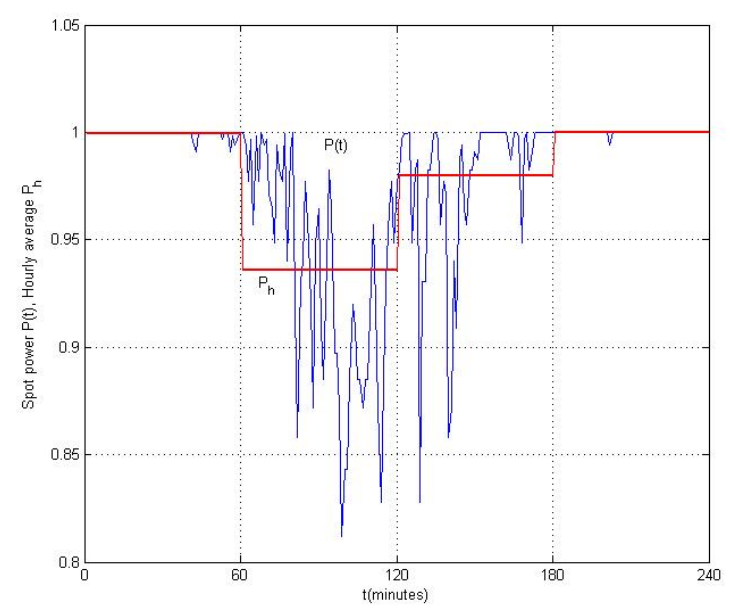

Fig. 3 Spot and hourly mean power in a wind generator.

The National Regulations of some countries with high RES penetration have defined some quality constraints for the divergences and its economical downsides. In this paper, we adopt a simplified model: the energy sent to the grid must meet some quality constraints if penalties are to be avoided. It must be in an offset band such as $P^{\prime}-\Delta \leq P o(t) \leq P^{\prime}+\Delta$. The $\Delta$ value is defined by the Grid Regulations, it can be defined as a fraction, $\delta$, of the nominal power: $\Delta=\delta P n$.

We define two logical conditions, the in band when the output power is within the offset band, $P o(t) \in P^{\prime} \pm \Delta$, and the converse out band condition when the output power is outside this offset band. We can introduce some measures of energy amount and quality. The raw energy provided by the RES generator $E_{\text {res }}$ and the energy feed in the grid $E_{\text {grid }}$ are defined as follows:

$$
E_{\text {res }}=\int P(t) d t \quad E_{\text {grid }}=\int P o(t) d t
$$

If no storage system is used, both values are the same. The planned energy, $E_{\text {plan }}$ and the energy fed into the grid outside of the quality band are expressed as: 


$$
E_{\text {plan }}=\int P^{\prime}(t) d t \quad E_{\text {out }}=\int_{P o(t) \notin P^{\prime} \pm \Delta} P o(t) d t
$$

We can introduce the excess or deficiency of energy feed when the system is out band as:

$$
E_{\text {deviation }}=\int_{P o(t) \notin P I \pm \Delta}\left|P o(t)-P^{\prime}\right| d t
$$

\subsection{Modeling the Storage System}

A simplified model of the storage subsystem is composed of two parts: the energy storage itself and the driver or set of physical devices(electronic, electrical and mechanical) that allows the storage and recovery processes. The driver subsystem is an abstract wrapper of a complex system involving very different technologies. The energy storage can be implemented by electric batteries or hydraulic reservoir, while the driver can be a system of power electronics or water turbines and pumps. We will suppose that the energy amount is an observable variable by mean of some suitable sensors. Let $E(t)$ and $E_{\max }$ be the stored energy and the maximum energy capacity of the storage subsystem, verifying: $0 \leq E(t) \leq E_{\max }$. The main issue in the modelling is the energy conservation equation. However, a detailed model is required to take account of the efficiency in the storage/recovery processes. The changes in the stored energy are defined as:

$$
\frac{d E}{d t}=\dot{E}_{\text {in }}-\dot{E}_{\text {out }}-\dot{E}_{\text {loss }}
$$

where $\dot{E}_{\text {in }}$ is the input rate in the storage phase, $\dot{E}_{\text {out }}$ is the rate in the energy recovery phase and $\dot{E}_{\text {loss }}$ is the rate of energy lost in the storage itself. The increase in the stored energy is the following when $E(t)<E_{\max }$

$$
\dot{E}_{\text {in }}=\left\{\begin{array}{cc}
\eta_{s}\left(P(t)-P^{\prime}\right) & P(t)>P^{\prime}+\delta_{1} \\
0 & \text { otherwise }
\end{array}\right.
$$

where $\eta_{s}$ is the efficiency of the driver in the storage phase, and $\delta_{1} \leq \Delta$. The decrease of energy in the recovery phase is the following when $E(t)>0$ :

$$
\dot{E}_{\text {out }}=\left\{\begin{array}{cc}
\left(1 / \eta_{r}\right)\left(P^{\prime}-P(t)\right) & P(t)<P^{\prime}-\delta_{2} \\
0 & \text { otherwise }
\end{array}\right.
$$

where $\eta_{r}$ is the efficiency of the driver in the recovery phase, and $\delta_{2} \leq \Delta$. It is possible to model some losses as a ratio of the stored energy:

$$
\dot{E}_{\text {loss }}=-P_{\text {loss }}-\lambda E(t)
$$

where $P_{\text {loss }}$ is a constant power of losses and $\lambda$ is a decay factor. The efficiency factors $\eta_{s}$ and $\eta_{r}$ in a hydraulic system are the efficiency of the pump in storage phase and the turbine in the recover one respectively. The output power that is sent to the grid, $P o(t)$, is:

$$
P o(t)=\left\{\begin{array}{cc}
P^{\prime} & P(t)>P^{\prime}+\delta_{1} \text { and } E(t)<E_{\text {max }} \\
P^{\prime} & P(t)<P^{\prime}-\delta_{2} \text { and } E(t)>0 \\
P(t) & \text { otherwise }
\end{array}\right.
$$

One additional constraint can be introduced by defining an upper value for the maximum gradient for energy change, $\left|\frac{d E}{d t}\right| \leq D_{\max }$, which is the maximum power of the driver system.

\section{Simulation}

We have designed a basic object to simulate storage related problems with limited upper and lower capacities. This basic object is related to the following differential equation involving $x(t)$ as the data, which is the rate of change of the stored value, and $y(t)$ which is the stored value itself:

$$
u(t)= \begin{cases}\eta_{s} x(t) & x(t) \geq 0 \\ \frac{1}{\eta_{r}} x(t) & x(t)<0\end{cases}
$$

$\frac{d y}{d t}+\lambda y=u(t) \quad y(t) \in\left[0, y_{\max }\right] \quad\left|\frac{d y}{d t}\right| \leq d_{\max }$

where $\lambda, y_{\max }, d_{\max }, \eta_{s}$ and $\eta_{r}$ are constants. Positive values of $x(t)$ mean increasing the $y(t)$ value, which constitutes a storage phase, while negative values of $x(t)$ mean decreasing the $y(t)$ value, which corresponds to recovery phase. Although it seems a linear system, it becomes nonlinear due to the constraints in the solution. This system has no solution for all values of $x(t)$. For example, if the storage is empty, $y=0$, and we try to recover at the rate value $x(t)$, which is impossible, it must be $\frac{d y}{d t}=0$ because it can not recover from an empty storage; the previous differential equation becomes: $0+\lambda 0=u(t)$, which means that only the value: $x(t)=0$ is a consistent solution in this case. We will distinguish between the $x(t)$ value as a request and $x^{\prime}(t)$ which is compatible, as an effective value. Despite the similarities, it is verified that: $x^{\prime}(t) \neq x(t)$ in the general case. To model some inertial in the storage and driver devices, we can introduce a low pass filter previous to the input as: $x(s) \rightarrow x(s) /(1+\tau s)$, where $\tau$ is a constant time value.

We have design a Simulink[8] block containing that generic storage module. Figure 4 shows the blocks of the modeling and simulation systems. The block Storage implements the defined model of a generic storage system focused on the power and energy management. The data source of the system is provided by the block windPower, which provides the spot power and some model of basic forecasting. It is implemented as a wrapper of a MATLAB file containing the power series in time steps of one minute and the whole series comprises 33 days. These data are obtained from wind speed series and a transfer function for a pitch regulated wind generator with values of $4 \mathrm{~m} / \mathrm{sec}$ and $13 \mathrm{~m} / \mathrm{sec}$ for cut-off and saturation respectively. The power is constant at the nominal value to the $25 \mathrm{~m} / \mathrm{sec}$ limit, which is never 


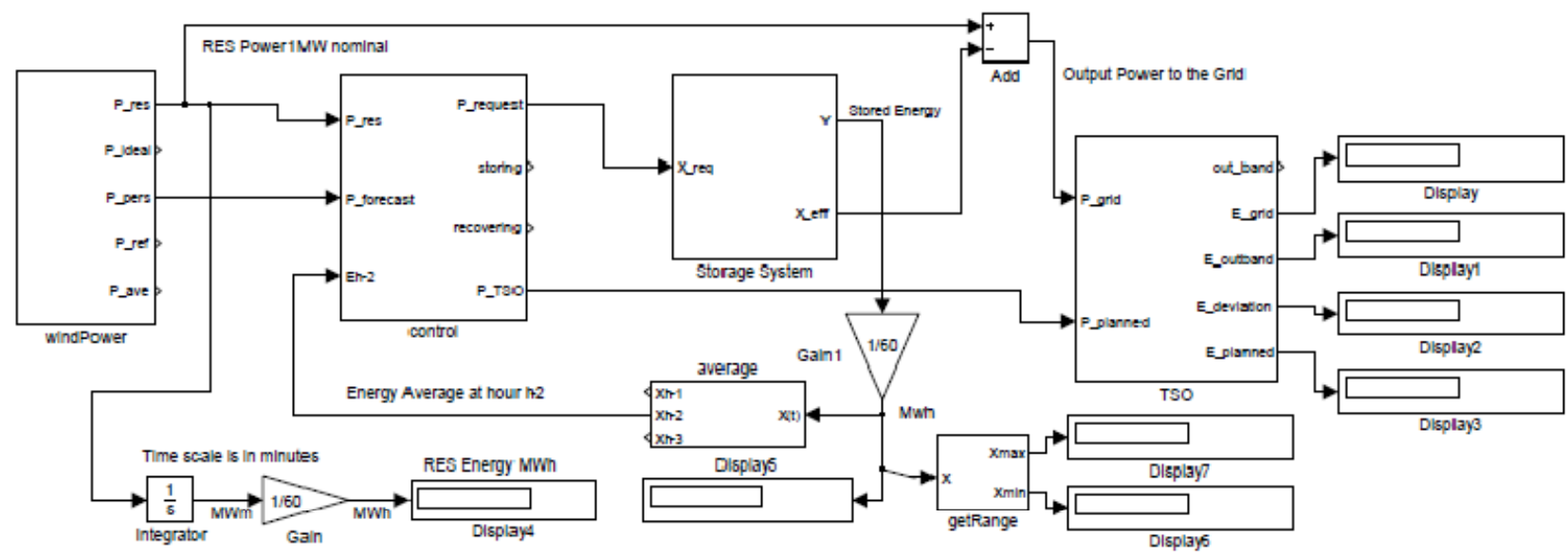

Fig. 4 Simulink blocks to test the storage system.

reached in the series. The block windPower also provides some values of three basic forecasting models for hourly periods. The simplest model is the persistence model, which provides the predicted value: $\hat{P}_{h+2}=P_{h}$. The second forecasting model is that suggested as the reference model[9,10], which provides the predicted values: : $\hat{P}_{h+2}=a_{2} P_{h}+\left(1-a_{2}\right) \bar{P}$, where $\bar{P}$ is a longterm average of the available data of source power and $a_{2}$ is the correlation coefficient between $P_{h}$ and $P_{h+2}$. These values in our case are: $a_{2}=0$ and $\bar{P}=0.68$. The last forecasting model is not actually a forecasting, we called it the ideal forecasting because is the best, and unreal, prediction that can be achieved: : $\hat{P}_{h+2}=P_{h+2}$. It is included only for testing purposes, because this ideal and unreal forecasting does not solve the problems concerning the lack of quality in the power fed to the grid.

By simulating the systems we have experienced that the storage system becomes systematically empty or full depending on the configuration parameters. In those states the system can neither store nor recover energy to regulate the output power, because it runs into its nonlinear zones. To avoid that the energy storage systematically becoming full or empty, a factor of innovation can be introduced in the planned power $k$ hours ahead as:

$$
\hat{P}_{h+k}^{(i n v)}=\hat{P}_{h+k}+k_{1}\left(E_{h}-E_{o b j}\right)
$$

where $E_{h}$ is the average stored energy in the $h$ hour, $k_{1}$ is a small constant parameter and $E_{o b j}$ is some objective level of storage. This strategy corrects the systematic bias and avoids the non linear states.

The Control block implements the storage strategy. An additional parameter has been added to avoid feeding power to the grid at power lower than a defined minimum value. This $P_{\min }$ value and the lower threshold $\delta_{2}$ means that no power is fed to the grid lower than the $P_{\min }-\delta_{2}$ value. It computes the planned power for each two hours ahead period and sends it to the TSO block. At every simulation step it computes the power balance and sends the requested power to the storage system to be stored or recovered. It uses the data provided by the Average block that implements the feedback innovation term to correct the states of bias.

The TSO block is mainly a logger of the power feed to the grid. It detects the in band and out band states according to the $\Delta$ parameter, which is defined in the Regulatory Norms of the Electricity Authority, and the planned power for each Market period. The energy feed in the different states is computed by integrating the power.

\section{Results}

The first test performed on the system was the computation of the results of the TSO block without any storage system. This test provided the raw quality factors corresponding to the RES generator. The test was based on a time series of 791 hours. The first three columns on Table 1, which have the label no storage(NS), contain the energy values for the three forecasting strategies, P(Persistence), R(Reference Model) and I(Ideal).

When the storage system is used, the energy provided by the RES generator is managed by the control system. It is stored and recovered according to the defined strategy. It means that some energy amount will be lost due to the efficiency of the storage driver. The use of the storage system provides more quality in the power fed to the grid, at the cost of lower amount of feed energy. The more quality, the less energy is an approach that will be economically feasible depending on the structure of prices, penalties and subsidies of each country.

Figure 4 shows the first 3900 minutes of the power provided by the RES generator. Figure 5 shows the planned power as is sent to the TSO 2 hours in advance. Figure 7 shows the power sent to the grid and Figure 8 shows the energy stored. The parameters for the control block are: $\delta_{1}=\delta_{2}=0.05, E_{o b j}=3 M W h$ and $P_{\text {min }}=$ $0.25 \mathrm{MWh}$. This means that no energy is fed with a power lower than $P_{\text {min }}-\delta_{2} P_{n}$. The parameters of the storage system are $E_{\text {init }}=3 \mathrm{MWh}, E_{\max }=5 \mathrm{MWh}$, $\lambda=0 . \eta_{s}=\eta_{r}=0.9$ and no constraint is imposed in the maximum allowable gradient. Figures show how the power holes of the RES generator are time-delayed in 
relation to the fed power. This allows the TSO to have the planned power two hours in advance, thus avoiding uncertainty in the planning of the public electricity system.

Table 1 contains the results for a large simulation, 791 hours, the same parameter previously considered with a lower efficiency: $\eta_{s}=\eta_{r}=0.8$, which means a global efficiency of $\eta_{s} \eta_{r}=0.64$. The columns without the label innovation(in) do not use the innovation factor, which means: $k_{1}=0$. Other included data are the values of the initial and final energy, as well as the maximum and minimum energy values.

In the columns without the innovation term, the Reference Model performs better than the other forecasting. It has the lowest values in out band and deviation energy. However, it was the more unstable because the storage became full and empty in the simulation. The last three columns have the best performance in quality. The storage was neither full nor empty, and also the final storage capacity was also close to the initial one. This means that the storage was always in the linear zone and the out band and deviation energies were null. However, the energy amount fed to the grid was lower in the three cases than in the same strategies in the previously considered groups.

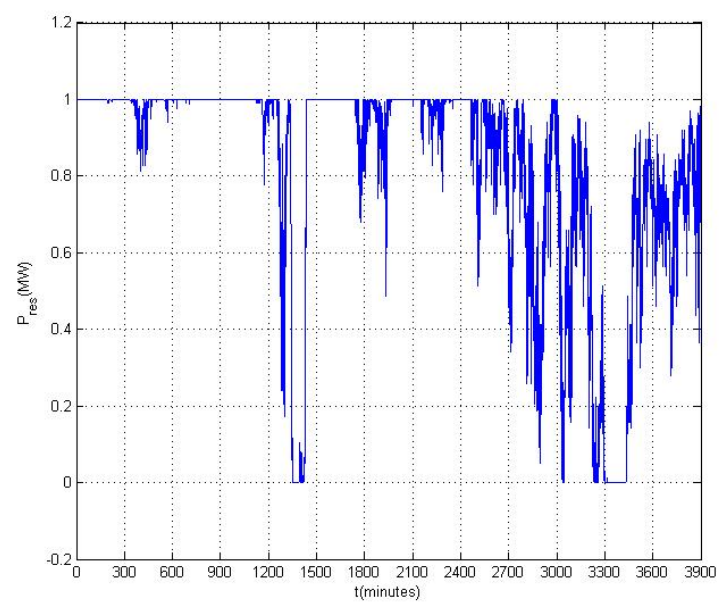

Fig. 5 RES power for the first 3900 minutes

\section{Conclusion}

The higher penetration of the RES in the future will introduce high disturbance into the electric systems by increasing the risk of instability. This risk can be avoided by increasing the spinning reserves; that is, by increasing the cost of the public electricity systems. The Electricity Regulations would move toward increasing the effects of the quality parameters in the system of prices and penalties. In addressing those problems, we have defined a mathematical model for energy storage based on general parameterized systems and also constructed a simulator focused on the management of the power and energy. This model can be used as a first level approach to simulate storage systems. With this approach, we avoid the device dependent details to obtain general conclusions about strategies, storage capacity, quality and efficiency. The simulator provides precise data about the increase in quality parameters and the corresponding decreasing in the amount of energy fed to the grid.

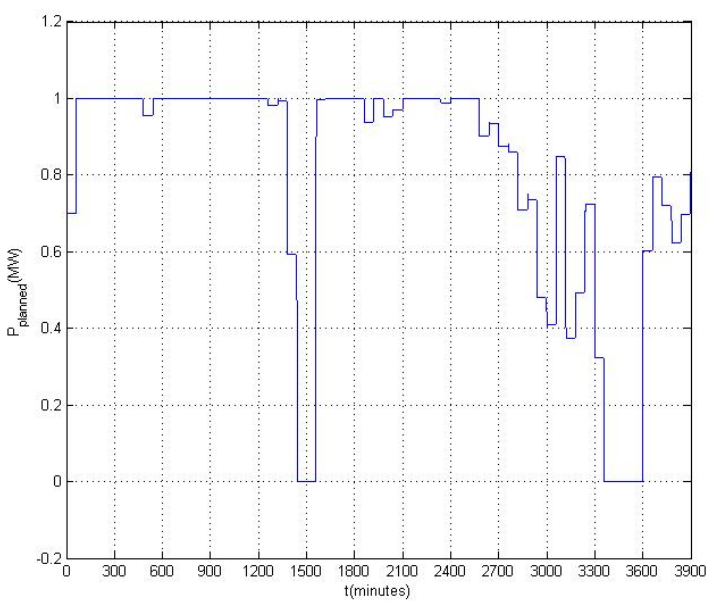

Fig. 6 Planned power sent to TSO

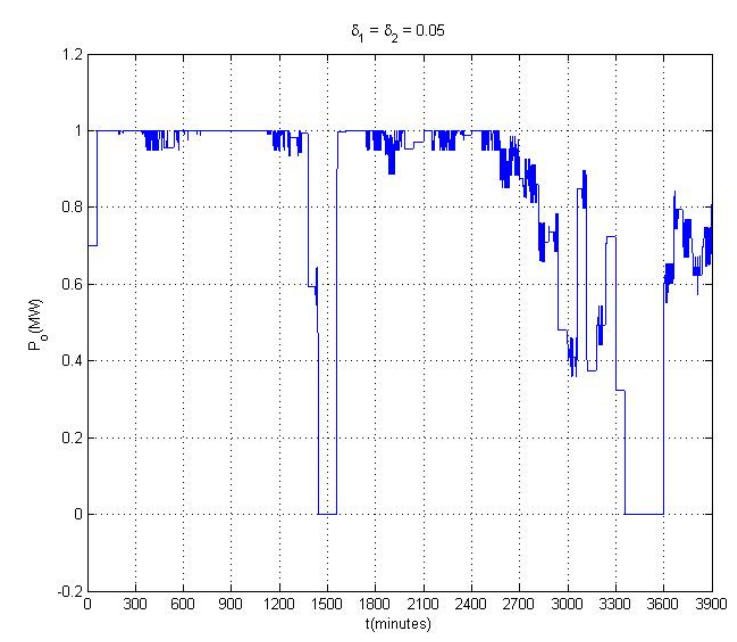

Fig. 7 Power fed to the grid fitting the quality requirements with at most $\pm 5 \%$ of the nominal power over the planned power

\section{Acknowledgment}

This research was partially supported by the Autonomous Government of Canary Islands, grant PI2007/039. The authors would like to thank to the Instituto Tecnológico de Canarias(ITC) for the use of the wind speed series acquired in its facilities in Pozo Izquierdo, Gran Canaria Island. 
Table 1 Quality parameters by using different forecasting strategies: P (persitence), $R$ (Reference model) and I(ideal), as well as NS (no storage) and (in )innovation filter.

\begin{tabular}{|c|r|r|r|r|r|r|r|r|r|}
\hline Energy(MWh) & $\mathrm{P}(\mathrm{NS})$ & $\mathrm{R}(\mathrm{NS})$ & $\mathrm{I}(\mathrm{NS})$ & \multicolumn{1}{l|}{$\mathrm{P}$} & \multicolumn{1}{l|}{$\mathrm{R}$} & $\mathrm{I}$ & $\mathrm{P}(\mathrm{in})$ & \multicolumn{1}{l|}{$\mathrm{R}(\mathrm{in})$} & $\mathrm{I}(\mathrm{in})$ \\
\hline$E_{\text {grid }}$ & 546.48 & 546.48 & 546.48 & 526.89 & 521.56 & 540.14 & 519.76 & 519.73 & 536.46 \\
\hline$E_{\text {out }}$ & 270.05 & 471.36 & 174.41 & 7.88 & 1.25 & 4.37 & 0.00 & 0.00 & 0.00 \\
\hline$E_{\text {deviation }}$ & 110.90 & 132.80 & 41.84 & 14.20 & 5.00 & 1.58 & 0.00 & 0.00 & 0.00 \\
\hline$E_{\text {plan }}$ & 546.51 & 546.63 & 546.48 & 540.93 & 525.81 & 540.90 & 519.68 & 518.67 & 534.78 \\
\hline$E_{\text {int }}$ & - & - & - & 3.00 & 3.00 & 3.00 & 3.00 & 3.00 & 3.00 \\
\hline$E_{\text {end }}$ & - & - & - & 0.43 & 2.11 & 0.01 & 2.95 & 3.32 & 2.86 \\
\hline$E_{\text {max }}$ & - & - & - & 3.00 & 5.00 & 3.42 & 3.52 & 3.59 & 3.48 \\
\hline$E_{\text {min }}$ & - & - & - & 0.00 & 0.00 & 0.00 & 0.73 & 0.92 & 2.45 \\
\hline
\end{tabular}

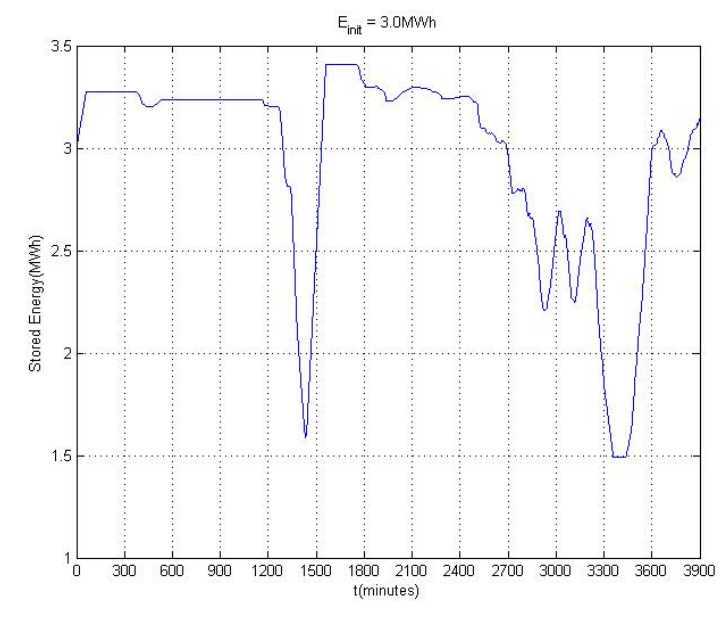

Fig. 8 Energy storage.

\section{References}

[1] I. Erlich, W. Winter and A. Dittrich, "Advanced requirements for the integration of wind turbines into the German transmission system” in IEEE Power Engineering Society General Meeting, 2006, pp 7-13.

[2] E. Panteri "EC project STORIES. Set of the existing regulations and legislative frameworks related to RES implementation”, http://www.storieproject.eu, 2008.

[3] P.B. Eriksen, T. Ackermann, H.H. Abildgaard, P. Smith, W. Winter and J.M.R. Garcia. "System operation with high wind penetration" in IEEE Power and Energy Magazine, Vol. 3, No. 6, pp 65-74, 2005.

[4] E.ON-Netz, "Wind Report 2004”, http://www.eonnetz.com, EON Tech Rep., 2004.

[5] S. Drouilhert, "Power flow management in a high penetration wind-diesel hybrid power system with short-term energy storage”, NREL Tech. Rep. CP-500-26827, July, 1999.

[6] J.O.G. Tande, E. Muljadi, O. Carlson, J. Pierik, A. Estanquiro, P. Sorensen, M. O’Malley, A. Mullane, O. AnayaLara and B. Lemstrom, "Dynamic models of wind farms for power systems studies”, IEA wind annex XXI, IEA Tech. Rep., 2007
[7] A.D. Hansen, P. Sorensen, F. Blaabjerg and J. Becho, "Dynamic modelling of wind farm grid interaction", Wind Engineering, Vol. 26, No. 4, pp 191-208, 2002.

[8] Simulink 7 Reference, The MathWorks Inc., 2008

[9] T. S. Nielsen, A. Joensen, H. Madsen, L. Landberg and G. Giebel, "A new reference model for wind power forecasting”, Wind Energy, vol. 1, pp 29-34, 1998.

[10] H. Madsen, "EC Project ANEMOS: A protocol for standardizing the performance evaluation of short-term wid power prediction models", Technical University of Demmark, Tech. Rep. 2004. 